\title{
Low-Frequency Sonoporation in vitro: Experimental System Evaluation
}

\author{
Jure Jelenc ${ }^{1}$ - Jože Jelenc ${ }^{1}$ - Damijan Miklavčič² - Alenka Maček Lebar2,* \\ 1 Iskra Medical d.o.o., Slovenia \\ 2 University of Ljubljana, Faculty of Electrical Engineering, Slovenia
}

\begin{abstract}
Sonoporation is a phenomenon where ultrasound increases cell membrane permeability. As the result, molecules that are otherwise deprived of transport mechanisms can be transported across the cell membrane. Several different experimental exposure systems are described in the literature. Low-frequency ultrasound $(<500 \mathrm{kHz})$ exposure systems can be divided into two groups: systems with the transducer directly immersed in the cell suspension and systems with the transducer in a water bath.

We developed an experimental system based on progressive ultrasound wave in a water bath. It consists of a transducer operating at $29.6 \mathrm{kHz}$ submerged in a water bath, and bath walls lined by ultrasound absorbing lining. Using a hydrophone, we evaluated ultrasound reflections inside the bath, both with and without acoustic lining on the bath's boundaries. We also built a finite element model of the system in order to calculate ultrasound parameters that are inaccessible by conventional hydrophone measurement due to equipment limitations. The experimental system will enable exposure of cells to pre-measured and pre-calculated ultrasound conditions.
\end{abstract}

Keywords: ultrasound, hydrophone, cavitation, finite element model

\section{O INTRODUCTION}

Sonoporation is a phenomenon where ultrasound increases cell membrane permeability. As a result, molecules that are otherwise deprived of transport mechanisms can be transported across the cell membrane. Transport through the membrane becomes possible for molecules with both small and large molecular mass [1] to [3]. If the cell remains capable of repairing the damage to the membrane and re-establishing a normal state, the phenomenon is called reversible sonoporation. If the cell dies as a consequence of ultrasound exposure, sonoporation is irreversible.

The mechanisms of in vitro sonoporation have not yet been fully explored, but are most often associated with cavitation. Cavitation is defined as the creation of new surfaces or expansion/contraction/distortions of pre-existing ones in a liquid [4] and [5], and is strongly depended on gas content of the liquid [6]. Cavitation occurs when a liquid is subjected to rapid changes of pressure; therefore ultrasound could be the reason for its appearance [4], [7] and [8]. Althought cavitation is usually related to gas bubbles in the extracellular fluid [9], recently cell intramembrane cavitation has been proposed [10]. However, to control the effects of sonoporation and connect them with the transient or stable cavitation [4], it is necessary to measure and control spatial and temporal ultrasound pressure.

Sonoporation was demonstrated using ultrasound waves of different frequencies. The most commonly used frequencies are those in therapeutic ( 1 to $3 \mathrm{MHz}$ ) and diagnostic ultrasound transducers (3 to $18 \mathrm{MHz}$ ) as well as lithotripsy transducers [11]. Nevertheless, several experiments have also been conducted with low-frequency ultrasound transducers (below $500 \mathrm{kHz}$ ).

Ultrasound frequencies above $1 \mathrm{MHz}$ have been used in a number of studies where fluorescent dyes, genetic material and chemotherapeutic drugs were efficiently delivered into cells [1] and [12] to [15]. Less research has been done on low-frequency sonoporation using frequencies below $500 \mathrm{kHz}$, which has also been demonstrated with dye delivery [16] to [19], genetic material delivery [11] and [20] and chemotherapeutic drug delivery into cells [21]. Regardless of the ultrasound frequency used in the sonoporation experiments; other ultrasound parameters needed for efficient sonoporation were reported inconsistently. Ultrasound pressure to which the cells are exposed is insufficiently reported; instead authors report on ultrasound intensity, energy or some other derived quantity [22].

Sonoporation studies are usually performed on cells suspended in the growth medium. Different types of experimental systems have been developed for research of low-frequency sonoporation. Depending on the way ultrasound waves are delivered to cells, these systems can be divided into two groups: systems where the ultrasound transducer and cells suspension are in direct contact and systems where dish containing the cell suspension is submerged in a water bath.

Exposure with direct contact between the ultrasound transducer and cell suspension is achieved by directly inserting the ultrasound transducer into the dish with the cell suspension. In addition to custom made ultrasound transducers, transducers for mixing and homogenizing mixtures in laboratory equipment 
have also been used for exposure of cells [23]. In this cylinder or horn-shaped transducer, the piezoelectric ultrasound transducer is located in the upper part. The ultrasound propagates along the cone or cylinder, with the tip of the object inserted into a dish with the cell sample. This method has been frequently used due to its simplicity, but has several disadvantages. The exposure of the cells in suspension to ultrasound is non-homogeneous because the ultrasound transducer is only inserted into part of the cell sample. Moreover, the small sample size does not allow insertion of ultrasound measurement devices into the cell sample. Furthermore, the sample can heat up quickly.

In the experiments with an ultrasound transducer submerged in water, ultrasound propagates through water to a submerged dish (usually a laboratory centrifuge tube) filled with a cell suspension. The main drawbacks of this method are possible ultrasound reflections caused by centrifuge tube, which are usually neglected as tubes are made of ultrasound transparent materials [24]. Heating of the cell suspension is minimized due to heat dissipation in water. A hydrophone inserted into the water bath allows ultrasound pressure inside the bath to be measured, and therefore the cells can be exposed to known, previously measured ultrasound pressure.

The most important parameter in sonoporation experimental system is ultrasound pressure, which is usually measured by a hydrophone. The hydrophone responds to acoustic waves generating a voltage proportional to the acoustic pressure [25]. The hydrophone measures the combination of the incident ultrasound pressure and the pressure due to reflections from the bath boundaries. The incident ultrasound can be measured directly either by using measurements of pulsed ultrasound or by avoiding reflections. Measurements of pulsed ultrasound allow ultrasound pressure to be measured before the reflected waves reach the hydrophone [25]. This approach does not reduce reflected waves, but simply allows us not to measure them. On the other hand, lining the walls of the water bath with an ultrasound absorber reduces the amount of reflected waves; therefore, only progressive ultrasound propagation is obtained. Such exposure conditions have been frequently used in sonoporation experiments [2], [20] and [26], but the amount of wave reflection reduction in sonoporation exposure systems using absorbing lining has not been reported.

The aim of the study was to design a system for observing influence of low frequency ultrasound in sonoporation in vitro, with well defined ultrasound pressure at the position of the cell sample. We constructed and evaluated a water bath experimental system based on progressive wave ultrasound propagation. The evaluation was performed by acoustic pressure measurements and by a finite element numerical model. Good agreement of measured and calculated pressure will allow us to report verified spatial and temporal ultrasound pressure characteristics, and relate them to the effects on the exposed cells.

\section{METHODS}

\subsection{Experimental System}

A water bath with a length of $68 \mathrm{~cm}$, width of $38 \mathrm{~cm}$ and height of $34 \mathrm{~cm}$ was filled with distilled water up to a height of $24 \mathrm{~cm}$ (Fig. 1). Ultrasound was generated using a prototype center bolt (Langevin type) piezoelectric ultrasound transducer [27] and [28] with an operating frequency of $29.6 \mathrm{kHz}$ (Iskra Medical, Slovenia). The transducer was submerged in a water bath at a depth of $12 \mathrm{~cm}$. Ultrasound pressure was measured with a piezoelectric hydrophone (8103 hydrophone, Brüel \& Kjær, Denmark) that was located on the central axis of the ultrasound transducer. Hydrophone positioning system allowed the distance between the hydrophone and transducer to be adjusted. The walls of the bath were made from Plexiglas ${ }^{\circledR}$. In some experiments the walls of the water bath were lined with the SA-J35 ultrasound absorber (Hangzhou Applied Acoustics Institute, China). According to the manufacturer this material decreases ultrasound reflection by $20 \mathrm{~dB}$ at a frequency of 30 $\mathrm{kHz}$.

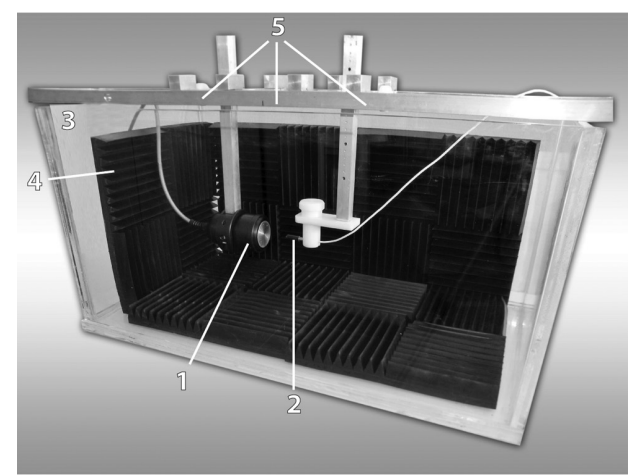

Fig. 1. Experimental system consisting of: an ultrasound transducer (1), a hydrophone (2), water bath walls (3), ultrasound absorbing lining (4) and a positioning system (5)

\subsection{Measuring Equipment}

Ultrasound pressure in the water bath was measured using a calibrated 8103 hydrophone (Brüel \& Kjær, 
Denmark). The active element of the hydrophone is a piezoelectric transducer. The 8103 hydrophone is designed for measuring pressure changes with frequencies between $0.1 \mathrm{~Hz}$ and $180 \mathrm{kHz}$. The sensitivity of the hydrophone to pressure changes as a function of the wave frequency was supplied by the manufacturer. We used the sensitivity value $0.08764 \mathrm{pC} / \mathrm{Pa}$, acquired at a frequency of $28 \mathrm{kHz}$. The hydrophone sensitivity changes by less than $0.5 \%$ when frequency increases up to $31.4 \mathrm{kHz}$. The hydrophone signal was amplified with a dedicated "Measuring amplifier 2525" (Brüel \& Kjær, Denmark). Real-time signal acquisition was performed by connecting the hydrophone output via the amplifier to a TPS 2024 oscilloscope (Tektronix, USA). Since the low-amplitude hydrophone signal may be distorted due to reflected waves or environmental disturbances, the signal acquisition was triggered by the voltage exciting the ultrasound transducer.

\subsection{Measuring the Ultrasound Pressure}

In order to study ultrasound reflection, we generated 8 periods of an ultrasound wave $(29.6 \mathrm{kHz}$, power set to $50 \%$ ) and measured the pressure with a hydrophone at a distance of $3.5 \mathrm{~cm}$ from the transducer. After measuring the amplitude of incident pressure over the first $0.6 \mathrm{~ms}$, we measured the reflected waves from 0.6 to $10 \mathrm{~ms}$. The measurement procedure was first performed in a bath with no absorbable lining. Then, the walls were lined with an absorber and the procedure was repeated. In both cases, the maximum amplitude of the signal was estimated. To evaluate the reduction of the reflection by the lining in the system, we evaluated an integral of absolute pressure from 0.6 to $10 \mathrm{~ms}$, which represents the area of the signal. The reduction is presented as a ratio of the area of the reflections measured with and without the absorbable lining.

The spatial distribution of ultrasound pressure was evaluated by measuring ultrasound at the axial center of transducer while varying the distance between the ultrasound transducer and hydrophone using a manual positioning system. The distance between the ultrasound transducer and hydrophone was varied from 1.5 to $10 \mathrm{~cm}$ in steps of $0.5 \mathrm{~cm}$. The walls of the bath were lined with the absorber. A continuous wave ultrasound $(\mathrm{cw})$ with power setting at $20 \%$ was used. The absolute measured value of peak compression was the same as the absolute value of peak rarefaction pressure. Root mean square pressure (RMS) is given as the mean and standard deviation of nine measurements, performed on 3 separate days.
Interday measurements were taken in order to take into account an error resulting from repositioning of the measuring system and changing the concentration of the dissolved gasses in the water. The nonlinear regression model was fitted to the measured data and the coefficient of determination $R^{2}$, was calculated using SigmaPlot 11 (Systat, USA).

\subsection{Finite Element Model}

The acoustic pressure generated in water by a composite Langevin type [27] sandwich transducer was modeled using finite element method (FEM) numerical calculations in Comsol 3.5 (Comsol Group, USA), by coupling structural mechanic, piezoelectric and pressure acoustic equations. The transducer's geometries (Fig. 2) were measured on a prototype transducer (Iskra Medical, Slovenia) that consists of two Lead Zirconate Titanate (PZT-4) elements sandwiched between the aluminum and the iron part. Three $0.2 \mathrm{~mm}$ adhesive layers were modeled between the metals and PZT-4 elements. The system was simplified by omitting the inner bolt and also the mechanical bias generated inside the transducer by the bolt. Material properties of the iron, two piezoelectric layers PZT-4, aluminum, and water were modeled using Comsol 3.5's material library. Additional three adhesive layers were described using Young's modulus (1010 Pa), Poisson's ratio (0.38) and density $\left(1700 \mathrm{~kg} / \mathrm{m}^{3}\right)$.

The tension inside the structural mechanic model was obtained using alternating current (AC) conditions. On each of the piezoelectric elements voltage electrical boundary condition was applied; amplitude was set on $260 \mathrm{~V}$ and frequency on $29.6 \mathrm{kHz}$. This voltage condition corresponds to the voltage measured on the transducer with power on the voltage generator set to $20 \%$. An electrical ground condition was applied to the other side of each piezoelectric element. The applied voltage induces mechanical stress $\sigma$ inside the piezoelectric material as described by equations:

$$
\begin{gathered}
\sigma=c_{E} \cdot \varepsilon-e^{T} \cdot E, \\
D=e \cdot \varepsilon+\varepsilon_{0} \varepsilon_{r S} \cdot E,
\end{gathered}
$$

where $\varepsilon$ is the strain vector, $c_{E}$ is the elastic stiffness matrix of the material under a constant electric field, $e$ is the piezoelectric stress matrix, $\varepsilon_{0} \varepsilon_{r s}$ is electric permittivity matrix under constant strain, $E$ is the electrical field vector and $D$ is the electrical displacement vector [29]. The stress and strain inside 
the transducer are calculated using the principle of virtual work [30]:

$$
\delta W=0 .
$$

Movement of the solid domain boundaries was unconstrained, but the displacement of the boundaries was considered small; thus, there was no need to remesh the model during calculation. The acceleration $a_{n}$ of the transducer-water boundary in the normal direction $n_{a}$ (outward-pointing from the water domain) is a source of pressure changes in water $p$, and $\nabla p$ is pressure gradient:

$$
n_{a} \cdot\left(\frac{1}{\rho} \cdot(\nabla p)\right)=a_{n} .
$$

The pressure $p$ in the water generates force $F_{n}$ on the water/solid boundary to the solid domain in the normal direction $n_{s}$ (outward-pointing from the solid domain):

$$
F_{n}=-n_{s} \cdot p .
$$

Ultrasound pressure in water was modeled using the homogeneous Helmholtz equation:

$$
\frac{1}{\rho \cdot c^{2}} \cdot \frac{\partial^{2} p}{\partial t^{2}}+\nabla \cdot\left(-\frac{1}{\rho} \cdot \nabla p\right)=0,
$$

where $p$ is pressure, $\rho$ is the density of water, and $c$ is the speed of sound in water. The equation was solved for a time-harmonic wave with an excitation frequency of $29.6 \mathrm{kHz}$. With arbitrary coordinate $x$, the pressure $p$ becomes periodic with an angular frequency $\omega$, and $i$ as imaginary unit:

$$
p(x, t)=p(x) \cdot e^{i \omega t} .
$$

Therefore, the homogeneous Helmholtz equation simplifies to:

$$
-\frac{\omega^{2} \cdot p}{\rho \cdot c^{2}} \cdot+\nabla \cdot\left(-\frac{1}{\rho} \cdot \nabla p\right)=0 .
$$

A perfectly matched layer was added on the far boundary of the liquid, which acts as a material where the loss factor increases, therefore damps the wave and does not cause any reflections on the boundary. Boundaries of the other materials are allowed to move without constraints.

To reduce the computational cost, the $3 \mathrm{D}$ model was reduced to a $2 \mathrm{D}$ axial symmetry model. The finite element mesh was generated with the maximum element size restricted to at least $1 / 5$ of the maximum wavelength. Due to the low computation cost of axial symmetry, and in order to achieve high precision, we used denser mesh consisting of $2 \cdot 10^{5}$ triangular elements.

\section{RESULTS}

\subsection{Measuring the Ultrasound Pressure}

The measured pulsed signal can be divided into two parts. The first 0.6 milliseconds represent eight pulses of incident ultrasound with maximum pressure amplitude of $135 \mathrm{kPa}$. Reflections of pulsed ultrasound from boundaries are shown in Fig. 3. Reflections with no acoustic lining are shown in Fig. $3 \mathrm{a}$, while reflections using the acoustic lining on the boundaries of the water bath are shown in Fig. 3b. In both cases, the measurement of the incident pressure causes measurement saturations. We have therefore omitted the first 0.6 seconds from Fig. 3. Fig. 3 thus only shows reflections. If no absorptive material is used, the maximum value of the reflected wave is 42 $\mathrm{kPa}$ or $31 \%$ of the incident pressure. The maximum value of reflection pressure using absorber is $7.8 \mathrm{kPa}$ or only $6 \%$ of the incident pressure. Use of the absorptive material reduces the area of the reflected wave signal to $7.8 \%$ of the area of the signal acquired without the use of the absorber.

The effect of the distance between the ultrasound transducer and hydrophone on the effective value of continuous-wave ultrasound pressure was observed at distances from 1.5 to $10 \mathrm{~cm}$. The largest effective value of ultrasound pressure was measured closest to the transducer at a distance of $1.5 \mathrm{~cm}: 111 \pm 10.6$ $\mathrm{kPa}$. Ultrasound pressure decreases exponentially as the distance between the transducer and hydrophone increases (Fig. 4). The standard deviation was considerably smaller within experiment done on the same day (less than 5\%), and increased when measurements from multiple days were pooled together (up to $15 \%$ ).

\subsection{Finite Element Model}

The calculated RMS pressure distribution in liquid induced by the transducer is shown in Fig. 2. In order to compare it to the measured results, we used results from the center of the axial symmetry (Fig. 4).

The maximum RMS pressure value calculated using the FEM model was at the transducer's boundary and had a value of $127 \mathrm{kPa}$. Experimental data at this distance was not accessible, due to spatial restrictions of our experimental setup. The first common measurement was therefore done at $1.5 \mathrm{~cm}$ from the 
transducer with a measured value of $111 \pm 10.6 \mathrm{kPa}$ and calculated value of $91 \mathrm{kPa}$.

Both results showed expected exponentially decaying RMS pressure value on the axial center of the transducer. Using nonlinear regression we fitted experimental results and FEM results onto a three parameters exponential decaying curve:

$$
p_{r}(d)=c_{1} \cdot e^{-c_{2} \cdot d}+c_{3},
$$

where $d$ is a distance between the transducer and the hydrophone. The regression parameters $c_{1}, c_{2}$ and $c_{3}$ and coefficient of determination $R^{2}$ are shown in Table 1.

Table 1. Nonlinear regression parameters

\begin{tabular}{ccccc}
\hline & $c_{1}[\mathrm{kPa}]$ & $c_{2}[1 / \mathrm{cm}]$ & $c_{3}[\mathrm{kPa}]$ & $R^{2}$ \\
\hline Experiment & 143.649 & 0.342 & 24.515 & 0.938 \\
\hline FEM & 120.387 & 0.338 & 17.984 & 0.999 \\
\hline
\end{tabular}

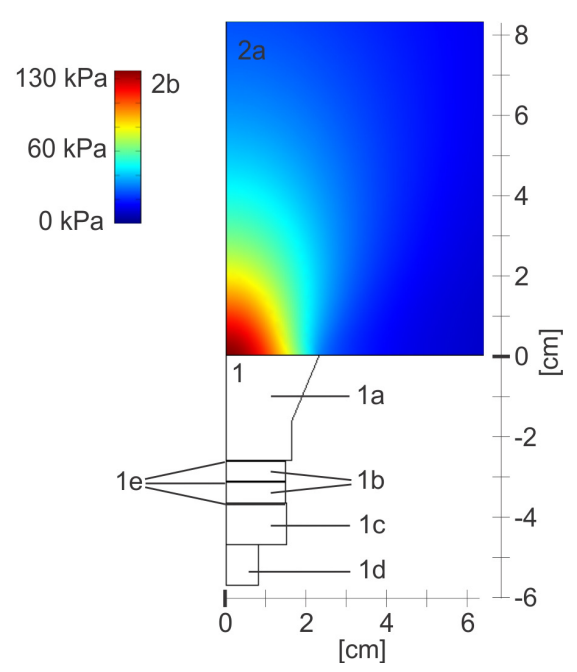

Fig. 2. Axial symmetry transducer geometry (1) and axial symmetry root mean square (RMS) pressure filed inside the water domain (2a), with a corresponding RMS pressure scale (2b); transducer geometry is composed of aluminum layer (1a), two piezoelectric layers (1b), iron layer (1c), bolt head made of iron (1d) and three narrow adhesive layers (1e); only one half of symmetry is shown
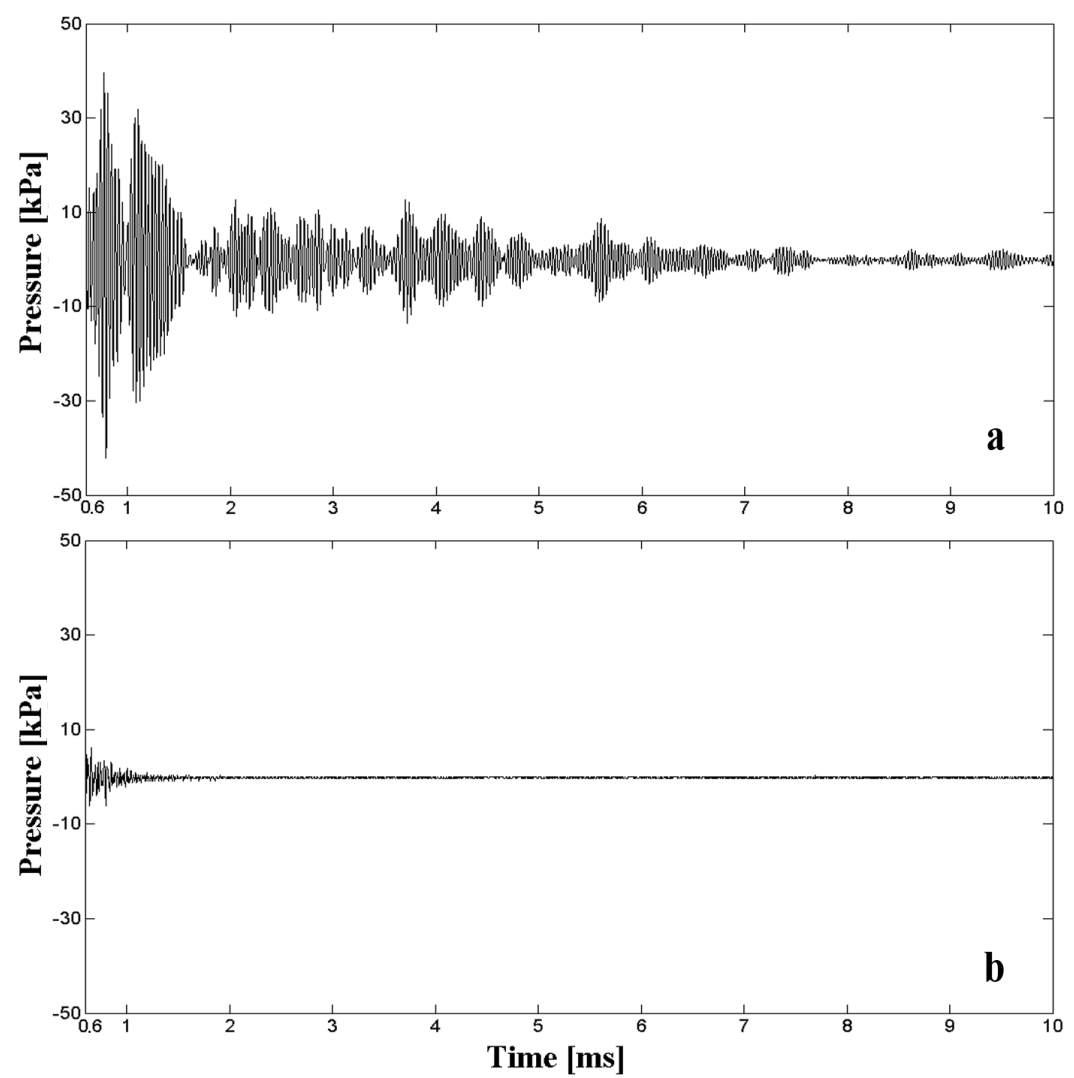

Fig. 3. Reflection of the eight periods of ultrasound signal from the boundaries; the reflections are shown 0.6 seconds after the signal was generated, so the incident ultrasound has already passed the hydrophone; signal a) without absorptive lining of the water bath walls and b) with the lining 


\section{DISCUSSION}

Even though a number of investigators have shown that cavitation, as the main mechanism of sonoporation, can more easily be induced by a standing wave than by a progressive wave [26] and [31], a standing wave is difficult to achieve in clinical settings due to variations of geometries inside the human body [31]. To increase the possibility of translation of the future results achieved in our in vitro exposure system to in vivo settings, we thus designed experimental system based on progressive wave ultrasound propagation.

As expected, the results of our study show that in an experimental system with finite dimensions reflected waves are present. Reflected waves are especially strong if no absorbable material is used. Though reflected waves are weaker than the waves coming directly from the ultrasound transducer, the pressure of the reflected waves in our system represents up to $31 \%$ of the pressure of the waves coming directly from the transducer. Reflected ultrasound waves are present in the water bath up to 2 $\mathrm{ms}$ after the ultrasound transducer has stopped emitting waves. At this time, ultrasound can travel across the longest dimension of the water bath four times. It is thus very likely that reflections of reflected waves are also present in the water bath. All these reflections are superimposed onto the incident pressure, thus preventing accurate measurements of the incident pressure. If the system is working continuously (cw), stationary waves may also occur and further reduce the accuracy of the incident pressure measurements. In our system, reflections were successfully reduced by lining the walls of the water bath with a special material that absorbs ultrasound. The area of the reflected wave signal was reduced by a factor of 12 , thus rendering the reflected waves barely noticeable (Fig. 3b).

To be able to expose cells to ultrasound in a water bath, we also need to determine spatial distribution of ultrasound waves in the bath. Therefore, we measured ultrasound pressure as a function of distance from the ultrasound transducer during continuous operation, in water bath with walls lined with ultrasound absorber. Measurements were performed at the transducer's central axis and showed an exponential decrease in ultrasound pressure as a function of distance. The standard deviation of the measurements was up to $15 \%$. This is a reasonable value considering we are dealing with a continuous (cw) low frequency ultrasound transducer. We have observed that intraday experiments had much smaller standard deviation of only a few percent. Some of the interday uncertainty can be associated to the positioning error of the hydrophone and the transducer and some to the change of water quality due to internalization of gasses from the air. The cavitation phenomenon could also alter ultrasound pressure in the water, but $120 \mathrm{kPa}$ ultrasound pressure is still considered to be a subcavitational level in a carefully processed water [20], and thus does not affect the measurements.

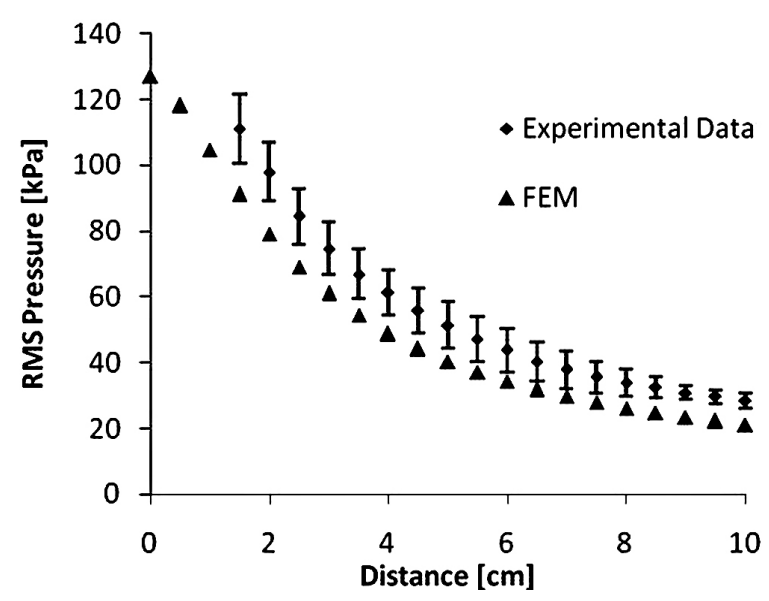

Fig. 4. The root mean square (RMS) value of ultrasound pressure at axial center of the transducer, as a function of distance between the transducer and hydrophone; the data was acquired using hydrophone measurements (diamonds) and calculation of FEM model (triangles); distance on transducer's central axis was varied from 1.5 to $10 \mathrm{~cm}$ in steps of $0.5 \mathrm{~cm}$

The results from the FEM model gave a good description of the system's behavior, although the calculated values were slightly smaller than those obtained experimentally. The FEM model results were affected by the model simplifications; mainly by omitting the inner bolt and the mechanical bias generated by the bolt. These were omitted due to a lack of relevant data needed for implementation into the FEM model. Bolt generated mechanical bias is known to increase ultrasound pressure amplitude [28]. Calculated values that do not incorporate mechanical bias thus must be lower than the measured ones. Also, some minor geometry features and a copper plate that serves as the electrical conductor for the PZT element have been omitted. When these limitations are resolved, the model accuracy could be further improved, especially the response at different frequencies.

By achieving good agreement between the hydrophone measurements and FEM model results we can conduct experiments at known ultrasound pressure anywhere in the experimental bath. In such a 
system, it is possible to expose biological materials to incident ultrasound under known pressure.

\section{CONCLUSIONS}

We have developed a system for low-frequency in vitro sonoporation experiments based on progressive ultrasound wave in water bath. It consists of a transducer submerged in a water bath, and bath walls lined by ultrasound absorbing lining. Using a hydrophone, we evaluated ultrasound reflections inside the bath, both with and without acoustic lining on the bath's boundaries. By using a specialized acoustic lining and sufficient size of the water bath, we were able to reduce the reflections to a level where measurements of low-frequency $(29.6 \mathrm{kHz})$ continuous wave ultrasound were made possible. In this regime, we evaluated the spatial characteristics of the system and confirmed them with a finite element model. This experimental system will enable exposure of cells to pre-measured and pre-calculated ultrasound conditions.

\section{ACKNOWLEDGMENTS}

Operation is part financed by the European Regional Development Fund (Biomedical Engineering Competence Center, Slovenia), European Social Fund and Slovenian Research Agency (L2-2044).

\section{REFERENCES}

[1] Liang, H.D., Tang, J., Halliwell, M. (2010). Sonoporation, drug delivery, and gene therapy. Proceedings of the Institution of Mechanical Engineers. Part H, Journal of Engineering in Medicine, vol. 224, no. 20, p. 343-361, DOI:10.1243/09544119JEIM565.

[2] Karshafian, R., Samac, S., Bevan, P.D., Burns, P.N. (2010). Microbubble mediated sonoporation of cells in suspension: clonogenic viability and influence of molecular size on uptake. Ultrasonics, vol. 50, no. 7, p. 691-697, DOI:10.1016/j.ultras.2010.01.009.

[3] Karshafian, R., Bevan, P.D., Burns, P.N., Samac, S., Banerjee, M. (2005). Ultrasound-induced uptake of different size markers in mammalian cells. IEEE Ultrasonics Symposium, p. 13-16, DOI:10.1109/ ULTSYM.2005.1602785.

[4] Leighton, T.G. (1997). The Acoustic Bubble. Academic Press, London.

[5] Neppiras, E.A. (1984). Acoustic cavitation series: part one: Acoustic cavitation: an introduction. Ultrasonics, vol. 22, no. 1, p. 25-28, DOI:10.1016/0041624X(84)90057-X.

[6] Dular, M., Širok, B., Stoffel, B. (2005). The influence of the gas content of water and the flow velocity on cavitation erosion aggressiveness. Strojniški vestnik Journal of Mechanical Engineering, vol. 51, no. 3, p. 132-145.

[7] Osterman, A., Dular, M., Širok, B. (2009). Numerical simulation of a near-wall bubble collapse in an ultrasonic field. Journal of Fluid Science and Technology, vol. 4, no. 1, p. 210-221, DOI:10.1299/ jfst.4.210.

[8] Osterman, A., Dular, M., Hočevar, M., Širok, B. (2010). Infrared thermography of cavitation thermal effects in water. Strojniski vestnik - Journal of Mechanical Engineering, vol. 56, no. 9, p. 527-534.

[9] Prentice, P., Cuschieri, A., Dholakia, K., Prausnitz, M., Campbell, P. (2005). Membrane disruption by optically controlled microbubble cavitation. Nature Physics, vol. 1, no. 2, p. 107-110, DOI:10.1038/nphys 148 .

[10] Krasovitski, B., Frenkel, V., Shoham, S., Kimmel, E. (2011). Intramembrane cavitation as a unifying mechanism for ultrasound-induced bioeffects. Proceedings of the National Academy of Sciences, vol. 108, no. 8, p. 3258-3263, DOI:10.1073/ pnas. 1015771108 .

[11] Wu, J., Nyborg, W.L.M. (2006). Emerging therapeutic ultrasound. World Scientific, Singapore, DOI:10.1142/9789812774125.

[12] Wells, D.J. (2010). Electroporation and ultrasound enhanced non-viral gene delivery in vitro and in vivo. Cell Biology and Toxicology, vol. 26, no. 1, p. 21-28, DOI:10.1007/s10565-009-9144-8.

[13] Newman, C.M.H., Bettinger, T. (2007). Gene therapy progress and prospects: ultrasound for gene transfer. I, vol. 14, no. 6, p. 465-475, DOI:10.1038/sj.gt.3302925.

[14] Miller, D.L., Pislaru, S.V., Greenleaf, J.E. (2002). Sonoporation: mechanical DNA delivery by ultrasonic cavitation. Somatic Cell and Molecular Genetics, vol. 27, no. 1-6, p. 115-134, DOI:10.1023/A:1022983907223.

[15] Postema, M., Gilja, O.H. (2007). Ultrasounddirected drug delivery. Current Pharmaceutical Biotechnology, vol. 8, no. 6, p. 355-361, DOI: $10.2174 / 138920107783018453$.

[16] Sundaram, J., Mellein, B.R., Mitragotri, S. (2003). An experimental and theoretical analysis of ultrasoundinduced permeabilization of cell membranes. Biophysical Journal, vol. 84, no. 5, p. 3087-3101, DOI:10.1016/S0006-3495(03)70034-4.

[17] Guzmán, H.R., Nguyen, D.X., Khan, S., Prausnitz, M.R. (2001). Ultrasound-mediated disruption of cell membranes. I. Quantification of molecular uptake and cell viability. The Journal of the Acoustical Society of America, vol. 110, no. 1, p. 588-596, DOI:10.1121/1.1376131.

[18] Guzmán, H.R., Nguyen, D.X., Khan, S., Prausnitz, M.R. (2001). Ultrasound-mediated disruption of cell membranes. II. Heterogeneous effects on cells. The Journal of the Acoustical Society of America, vol. 110, no. 1, p. 597-606, DOI:10.1121/1.1376130. 
[19] Keyhani, K., Guzmán, H.R., Parsons, A., Lewis, T.N., Prausnitz, M.R. (2001). Intracellular drug delivery using low-frequency ultrasound: quantification of molecular uptake and cell viability. Pharmaceutical Research, vol. 18, no. 11, p. 1514-1520, DOI:10.1023/A:1013066027759.

[20] Wei, W., Zheng-zhong, B., Yong-jie, W., Qing-wu, Z., Ya-lin, M. (2004). Bioeffects of low-frequency ultrasonic gene delivery and safety on cell membrane permeability control. Journal of Ultrasound in Medicine, vol. 23, no. 12, p. 1569-1582.

[21] Tachibana, K., Uchida, T., Tamura, K., Eguchi, H., Yamashita, N., Ogawa, K. (2000). Enhanced cytotoxic effect of Ara-C by low intensity ultrasound to HL-60 cells. Cancer Letters, vol. 149, no. 1-2, p. 189-194, DOI:10.1016/S0304-3835(99)00358-4.

[22] ter Haar, G., Shaw, A., Pye, S., Ward, B., Bottomley, F., Nolan, R., Coady, A.-M. (2011). Guidance on reporting ultrasound exposure conditions for bio-effects studies. Ultrasound in Medicine \& Biology, vol. 37, no. 2, p. 177-183, DOI:10.1016/j.ultrasmedbio.2010.10.021.

[23] Pong, M., Umchid, S., Guarino, A.J., Lewin, P.A., Litniewski, J., Nowicki, A., Wrenn, S.P. (2006). In vitro ultrasound-mediated leakage from phospholipid vesicles. Ultrasonics, vol. 45, no. 1-4, p. 133-145, DOI:10.1016/j.ultras.2006.07.021.

[24] Kaddur, K., Lebegue, L., Tranquart, F., Midoux, P., Pichon, C., Bouakaz, A. (2010). Transient transmembrane release of green fluorescent proteins with sonoporation. IEEE Transactions on Ultrasonics, Ferroelectrics, and Frequency Control, vol. 57, no. 7, p. 1558-1567, DOI:10.1109/TUFFC.2010.1586.
[25] IEC 62127-1 (2007). Ultrasonics - Hydrophones - Part 1: Measurement and characterization of medical ultrasonic fields up to $40 \mathrm{MHz}$. International Electrotechnical Commission, Geneva.

[26] Kinoshita, M., Hynynen, K. (2007). Key factors that affect sonoporation efficiency in in vitro settings: the importance of standing wave in sonoporation. Biochemical and Biophysical Research Communications, vol. 359, no. 4, p. 860-865, DOI:10.1016/j.bbrc.2007.05.153.

[27] Radmanovic, M.D., Mancic, D.D. (2004). Design and modeling of the power ultrasonic transducers, University of Niš, Niš.

[28] Moreno, E., Acevedo, P., Fuentes, M., Sotomayor, A., Borroto, L., Villafuerte, M.E., Leija, L. (2005). Design and construction of a bolt-clamped Langevin transducer. International Conference on Electrical and Electronics Engineering, Proceedings, p. 393-395, DOI:10.1109/ICEEE.2005.1529652.

[29] Abboud, N.N., Wojcik, G.L., Vaughan, D.K., Mould, J., Powell, D.J., Nikodym, L. (1998). Finite element modeling for ultrasonic transducers. Proceedings of the SPIE International Symposium on Medical Imaging, p. 19-42.

[30] Szabó, B.A., Babuska, I. (1991). Finite element analysis. John Wiley \& Sons, New York.

[31] Barati, A.H., Mokhtari-Dizaji, M., Mozdarani, H., Bathaie, Z., Hassan, Z.M. (2007). Effect of exposure parameters on cavitation induced by low-level dualfrequency ultrasound. Ultrasonics Sonochemistry, vol. 14, no. 6, p. 783-789, DOI:10.1016/j. ultsonch.2006.12.016 